\title{
Reduced-error constant correction truncated multiplier
}

\author{
Dong Wang ${ }^{1 \mathrm{a})}$, Peng $\mathrm{Cao}^{2}$, and Yang Xiao ${ }^{1}$ \\ ${ }^{1}$ Institute of Information Science, Beijing Jiaotong University \\ ${ }^{2}$ National ASIC System Engineering Technology Research Center, \\ Southeast University \\ a)wangdong@bjtu.edu.cn
}

Abstract: Constant correction truncated multiplier can minimize hardware cost and power dissipation by computing only the most significant bits of partial products. However, traditional schemes introduce large truncation errors that degenerate the accuracy of the target application. In this brief, we propose an improved scheme that can significantly reduce such truncation errors. The proposed scheme estimates an accurate compensating constant by correctly counting the probability of occurrence of the input operand and the product bits with a group of probability propagation formulas. The proposed truncated multiplier is coded in Verilog RTL, implemented in $65 \mathrm{~nm}$ standard cell technology, and applied in image compression and color space conversion applications. Experimental results show that our scheme achieves an average peak signal-to-noise ratio (PSNR) improvement of $4.09 \mathrm{~dB}$ and $2.31 \mathrm{~dB}$ over state-of-the-art truncation scheme in the two applications, respectively. When the same truncation error range is desired, the proposed scheme can save around $5.8 \%$ of circuit area.

Keywords: constant correction truncated multiplier, digital signal processing

Classification: Electron devices, circuits, and systems

\section{References}

[1] H. J. Ko and S. F. Hsiao: IEEE Trans. Circuits Syst. II 58 (2011) 304. DOI:10.1109/TCSII.2011.2148970

[2] M. J. Schulte and E. E. Swartzlander: Proc. IEEE Workshop VLSI Signal Process. (1993) 388.

[3] C. H. Chang and R. K. Satzoda: IEEE Trans. VLSI Syst. 18 (2010) 1767. DOI:10.1109/TVLSI.2009.2027327

[4] E. J. King and E. E. Swartzlander: Proc. Asilomar Conference on Signals, Systems \& Computers (1997) 1178. DOI:10.1109/ACSSC.1997.679090

[5] M. G. Solaz, W. Han and R. Conway: IEEE Trans. Circuits Syst. I 59 (2012) 2555. DOI:10.1109/TCSI.2012.2189059

[6] V. Mahalingam and N. Ranganathan: Proc. VLSI Design (2006) 6. DOI:10. 1109/VLSID.2006.42

[7] M. G. Solaz, W. Han and R. Conway: IEEE Trans. Circuits Syst. I 59 (2012) 2555. DOI:10.1109/TCSI.2012.2189059 
[8] E. G. Walters, III, M. G. Arnold and M. J. Schulte: SPIE Proc. Advanced Signal Processing Algorithms, Architectures, and Implementations XIII (2003) 573.

[9] V. P. Hoang and C. K. Pham: IEICE Trans. Fundam. Electron. Commun. Comput. Sci. E95-A [6] (2012) 999. DOI:10.1587/transfun.E95.A.999

[10] S. Yu and E. E. Swartziander: IEEE Trans. Comput. 50 (2001) 985. DOI:10. $1109 / 12.954513$

\section{Introduction}

Multiplication is a fundamental arithmetic operation that is pervasively used in digital signal processing (DSP) applications, such as filtering, convolution and compression. From a hardware implementation perspective, an $n \times n$-bit multiplication yields a $2 n$-bit product. To maintain the full accuracy of the system, a DSP architecture requires an ever-growing bit width that would be impossible or impractical to implement. One commonly used method is truncating and rounding the results with the $n$ most significant bits so that they fit the limits of the architectural bit width [1]. The circuit area and dynamic power dissipation can also be reduced proportionally.

Several truncation schemes have been proposed for parallel digital multiplier designs $[2,3,4,5]$. Schulte et al. introduced the constant correction truncated $(\mathrm{CCT})$ scheme, in which $(n-k)$ columns of partial products are truncated, where $k$ is a nonnegative integer between 0 and $n-1$, and a nonzero constant is added to the final result to compensate the expected value of the truncation error. In [3], Chang et al. extended the CCT scheme for multiplexor based array multiplier and introduced adaptive pseudo-carry to compensate the truncation error (PCT), which resulted in a carry-saved multiplexor array structure. In [4], King et al. proposed a variable correction scheme (VCT) which used information from the partial product bits of the column adjacent to the truncated parts to better estimate the truncation error. Solaz et al. [5] presented a full precision multiplier which was implemented in a manner that the effective partial product matrix is selected dynamically at run-time. This scheme allows a power reduction tradeoff against signal degradation which can be modified at run-time. Software compensation of truncation errors was used in this scheme. Other hybrid strategies $[6,7]$ have also been proposed in the literature.

In the standard CCT scheme [2], the value of the compensating constant is estimated by the expected values of the reduction and rounding errors. We assume that multiplication of two $n$-bit numbers $A$ and $B$ results in a $2 n$-bit product $P$ with the forms

$$
A=\sum_{i=0}^{n-1} a_{i} \cdot 2^{-n+i}, \quad B=\sum_{i=0}^{n-1} b_{i} \cdot 2^{-n+i}, \quad P=\sum_{i=0}^{2 n-1} p_{i} \cdot 2^{-2 n+i}
$$

where $a_{i}$ and $b_{i}$ denote each bit of $A$ and $B$, and $p_{i}$ denotes each bit of $P$, respectively. The relationship between the partial product bit $\pi_{i, j}=a_{i} \cdot b_{j}$ and the final product bit $p_{i}$ in the multiplication matrix is shown in Fig. 1-a. The 


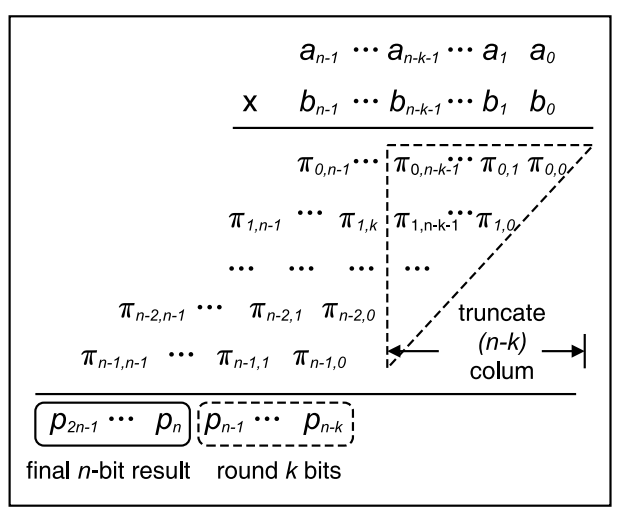

(a)

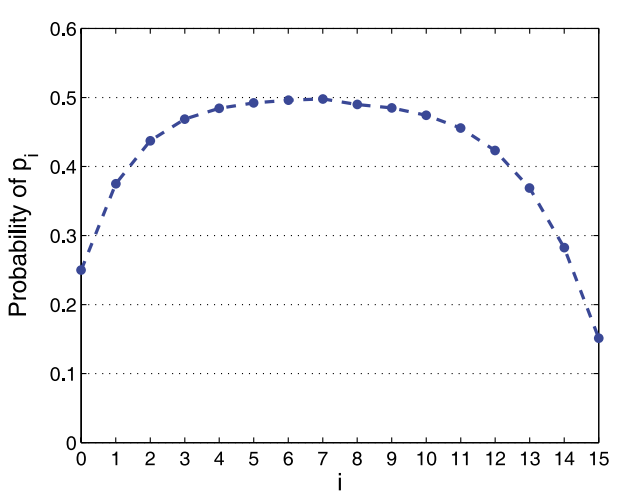

(b)

Fig. 1. (a) The partial product matrix of a $n \times n$-bit multiplier. (b) The measured probability distribution of the final product bits $p_{i}(n=8)$.

least significant $(n-k)$ columns of partial products are truncated and only the $(n+k)$ most significant columns are summed. Typically, the final product is also rounded to $n$ bits to avoid continuous growth of the word-length.

The expected value (i.e., the statistical average value) of the reduction error, which is the sum of all partial products in columns 0 to $(n-k-1)$, is calculated as

$$
E_{\text {reduct }}=-\frac{1}{4} \cdot \sum_{j=0}^{n-k-1}(j+1) \cdot 2^{-2 n+j}
$$

whereas the expected value of the rounding error is estimated by

$$
E_{\text {round }}=-\frac{1}{2} \cdot \sum_{j=n-k}^{n-1} 2^{-2 n+j}=-2^{-n-1} \cdot\left(1-2^{-k}\right)
$$

The expected value of the total truncation error $E_{\text {total }}$ is the sum of $E_{\text {reduct }}$ and $E_{\text {round }}$, and consequently, the correction constant is selected as the inverse value of the total error with $(n+k)$ bits precision, which is given as

$$
C=-\operatorname{rnd}\left(2^{n+k} \cdot E_{\text {total }}\right) \cdot 2^{-(n+k)}
$$

where function $\operatorname{rnd}(x)$ rounds $x$ to its nearest integer. From a statistical perspective, the standard CCT scheme is expected to minimize the average error of the final products.

However, two incorrect assumptions are made in deriving (2) and (3). It is assumed that both $a_{i}$ and $b_{i}$ have an equal probability of being "1" or "0", which leads to the conclusion that the probability of the partial product bit $\pi_{i j}$ being one is always $1 / 4$ in (2). Unfortunately, in real-world applications, the probability of the input bits $a_{i}$ and $b_{i}$ are typically not evenly distributed. One good example is the discrete cosine transform (DCT) algorithm for digital image compression [8]. In the DCT formula, each 8-bit pixel value (as input $A$ ) is multiplied by a transform coefficient (as input $B$ ), which is usually an 8-bit integer constant. Obviously, the probability of $b_{i}$ being one does not always equal to $1 / 2$. For instance, given $B=(113)_{10}=\left(1110 \_0001\right)_{2}, b_{i=7,6,5,0}$ 
always equal one. Therefore, using formula (2) leads to an incorrect estimation of the reduction error.

It is also assumed in (3) that the probability of each product bit, from $p_{n-1}$ to $p_{n-k}$, being one is $1 / 2$. As shown in Fig. 1 -b, a real-valued simulation is conducted to measure the actual probability of each product bit $p_{i}$. The input bits $a_{i}$ and $b_{i}$ are set with the same probability of $1 / 2$ to be one. The result shows that this assumption is also incorrect.

In this paper, we propose an improved scheme to correctly compute the probability of the partial and final product bits based on the actual probability of occurrence of input operand bits. By using this scheme, we can accurately estimate the compensating constant, and therefore, reduce the computation error introduced by truncation and rounding.

\section{Operand probabilistic estimation-based CCT multiplier}

For a $n \times n$-bit multiplication, the partial product matrix of Fig. 1-a can be implemented as an array multiplier consisting of half and full adders as shown in Fig. 2. Denoting $p\left(b_{i}\right)$ and $p\left(a_{j}\right)$ as the probability of the input bits $b_{i}$ and $a_{j}$ being one and $p(i, j)$ as the probability of the partial product bit $\pi_{i, j}$ at column $i$ and row $j$ being one, we know straightforwardly that $p(i, j)=p\left(b_{i}\right) \cdot p\left(a_{j}\right)$. However, the probability of $p_{i}$ can not be obtained directly. We propose using the following probability propagation formulas to compute $p(i)$. First, we denote $p_{s}(i, j)$ and $p_{c}(i, j)$ as the probability of the sum and carry bits of the adder at the $i$-th column and $j$-th row being one, respectively. As the partial products are accumulated by the adders from the upper-left corner of the array to the bottom-right corner, $p_{s}(i, j)$ and $p_{c}(i, j)$ can be calculated in a row-by-row manner. For $i=1$ and $0 \leq j \leq n-2$ (the second row of the product array), the following equations are used

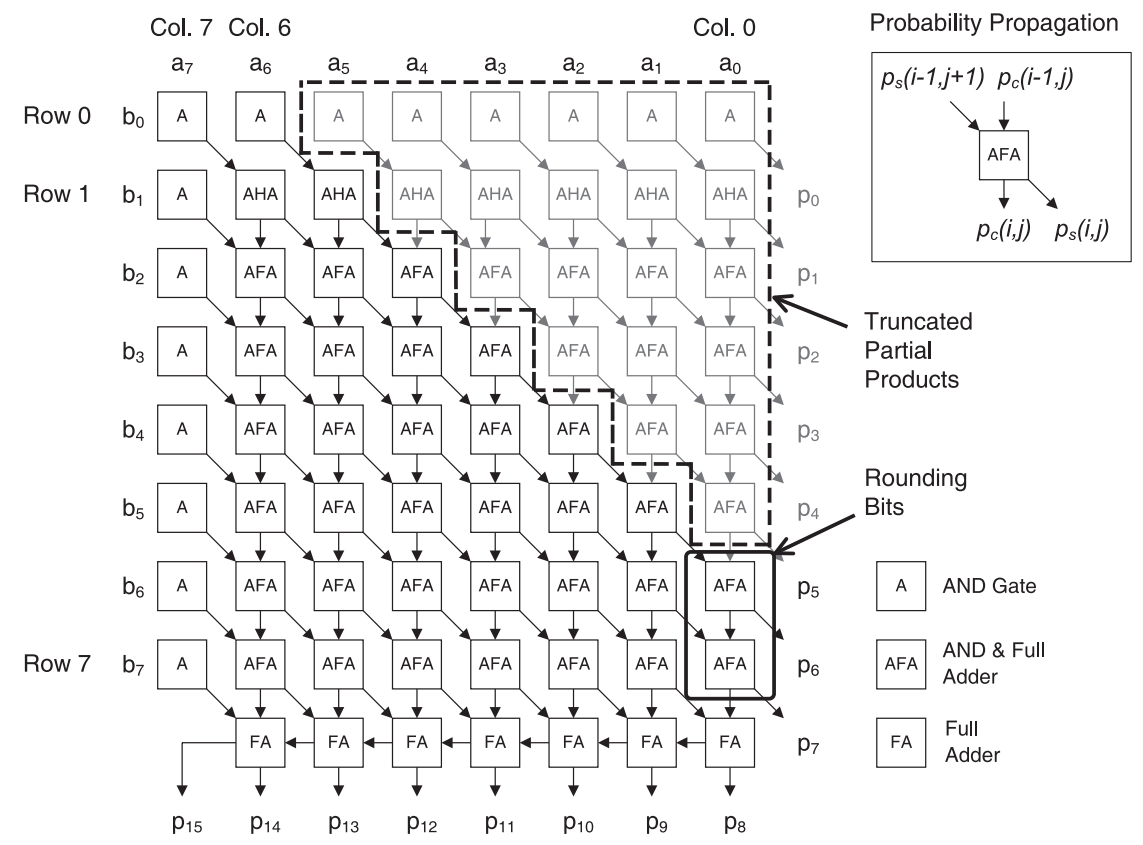

Fig. 2. Block diagram of a $n \times n$-bit truncated array multiplier $(n=8, k=2)$. 


$$
\begin{gathered}
p_{s}(1, j)=p(1, j) \cdot q(0, j+1)+q(1, j) \cdot p(0, j+1) \\
p_{c}(1, j)=p(1, j) \cdot p(0, j+1)
\end{gathered}
$$

where $q(i, j)=1-p(i, j)$, which represents the probability of the partial product $\pi_{i, j}$ being zero.

For $2 \leq i \leq n-1$ and $0 \leq j \leq n-2$, the following equations are used

$$
\begin{aligned}
p_{s}(i, j)= & p(i, j) \cdot p_{s}(i-1, j+1) \cdot p_{c}(i-1, j) \\
& +p(i, j) \cdot q_{s}(i-1, j+1) \cdot q_{c}(i-1, j) \\
& +q(i, j) \cdot q_{s}(i-1, j+1) \cdot p_{c}(i-1, j) \\
& +q(i, j) \cdot p_{s}(i-1, j+1) \cdot q_{c}(i-1, j) \\
p_{c}(i, j)= & p(i, j) \cdot p_{s}(i-1, j+1) \cdot p_{c}(i-1, j) \\
& +p(i, j) \cdot p_{s}(i-1, j+1) \cdot q_{c}(i-1, j) \\
& +p(i, j) \cdot q_{s}(i-1, j+1) \cdot p_{c}(i-1, j) \\
& +q(i, j) \cdot p_{s}(i-1, j+1) \cdot p_{c}(i-1, j)
\end{aligned}
$$

where $q_{s}(i, j)=1-p_{s}(i, j)$ and $q_{c}(i, j)=1-p_{c}(i, j)$. For $j=n-1, p_{s}(i, j)=$ $p(i, j)$. We note that $p(i)=p_{s}(i, 0)$. Consequently, for probability distribution parameters of $p\left(b_{i}\right)$ and $p\left(a_{j}\right)$ of given input operands, the $p(i)(0 \leq i \leq 7)$ can be accurately calculated by using the probability propagation formulas from (5) to (8). Therefore, we obtain the new expected value of the reduction error as

$$
E_{\text {reduct }}^{\prime}=-\sum_{i=0}^{n-k-1} \sum_{j=0}^{i} p(i-j, j) \cdot 2^{-2 n+i}
$$

and the expected value of the rounding error, which is the sum of products $p_{n-1}$ to $p_{n-k}$, as

$$
E_{\text {round }}^{\prime}=-\sum_{i=n-k}^{n-1} p_{s}(i, 0) \cdot 2^{-2 n+i}
$$

Then, similar to the error in the standard CCT scheme, the total truncation error is $E_{\text {total }}^{\prime}=E_{\text {reduct }}^{\prime}+E_{\text {round }}^{\prime}$, and the new correction constant $C^{\prime}$ can be calculated by using (4).

In the proposed scheme, the value of the compensating constant $C^{\prime}$ is determined by the actual probability of the input operands. Therefore, the expected value of the computation errors are closer to zero than they are in traditional schemes. To verify the effectiveness of the proposed operand probabilistic estimation-based (OPEB) error compensation scheme, we performed software based simulations for $8 \times 8$-bit and $16 \times 16$-bit truncated multiplications with different configurations of $k$. The recorded average truncation errors $E_{\text {total }}$ (expected values) are reported in Figs. 3 and 4 . For the $8 \times 8$-bit multiplication, we chose $p\left(b_{i}\right)=p\left(a_{j}\right)=1 / 2$ for $i=0, \cdots, 7$ (i.e., both are random inputs), whereas for the $16 \times 16$-bit multiplication, we changed the probability of operand $B$ as $p\left(b_{i}\right)=1$ for $i=3,7,8,10,12,15$ and $p\left(b_{i}\right)=0$ for other $i$ (i.e. operand $B$ is a constant). It can be observed that the proposed scheme achieves considerably error reductions over the 


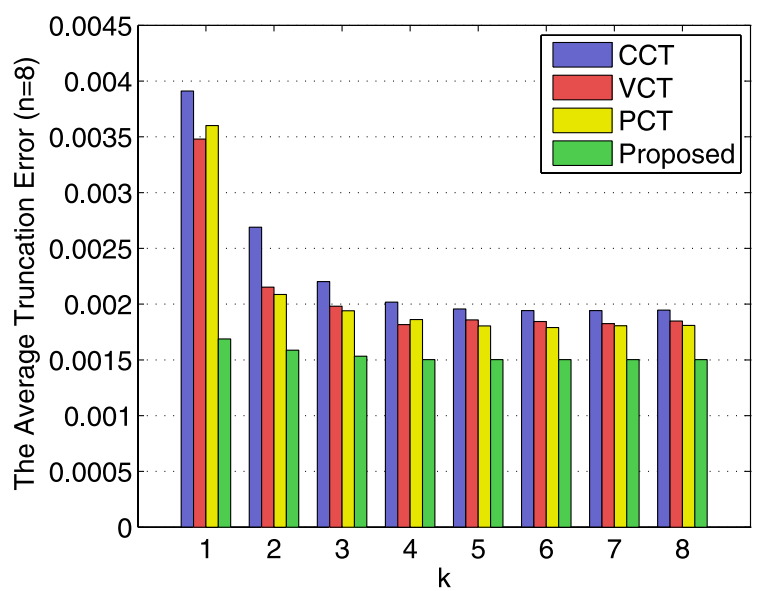

Fig. 3. Comparison of the averaged truncation errors between CCT, VCT, PCT and the proposed scheme for $n=8$.

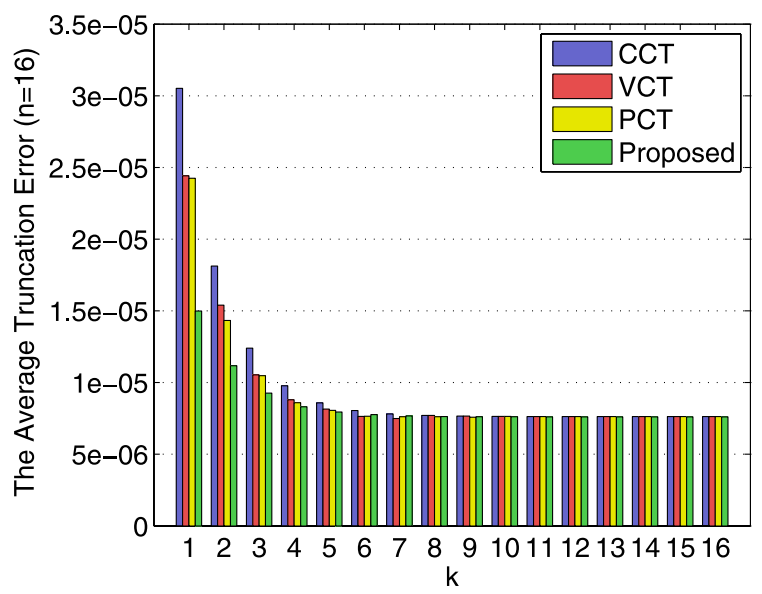

Fig. 4. Comparison of the averaged truncation errors between CCT, VCT, PCT and the proposed scheme for $n=16$.

three reference schemes when $k$ is smaller than 3 . The highest error reduction rate is $50.8 \%, 38.6 \%$ and $38.1 \%$, when $n=16, k=1$, respectively. Note that for very large $k$, all the schemes have close errors because only minimal parts of the productions are truncated. Hardware implementation results are presented in the following section.

\section{Implementation results and comparisons}

The proposed OPEB-CCT scheme is applied to design truncated array multipliers (Fig. 2-a). Four $16 \times 16$-bit multipliers configured with $k=1,2,3,4$ were coded in Verilog HDL, functionally verified by Cadence NC-Sim 5.1 and implemented in TSMC $65 \mathrm{~nm}$ CMOS standard cell technology. The target working frequency is $100 \mathrm{MHz}$ at $1.2 \mathrm{~V}$. Table I reports the measured area, which is normalized to the equivalent gate count (i.e., total area versus the area of a 2-input NAND gate). Three most frequently cited schemes, including 
CCT, VCT and PCT, have also been implemented and compared in the table. For each of reference designs, the parameter $k$ is selected with the smallest value which makes the truncation error $E_{\text {total }}$ closest to that of the OPEBCCT multiplier. $E_{\text {total }}$ is normalized in terms of the unit in the last place (ulp) of the output result. The result shows that the proposed scheme achieves approximately $10 \%, 7.9 \%$ and $5.8 \%$ area reductions compared to the CCT, VCT and PCT approaches, respectively. Our scheme can estimate the truncation error more accurately, and therefore, uses a smaller $k$, which results in a hardware structure that requires fewer adders.

Table I. Area (gate count) comparison between CCT, VCT, PCT and the proposed scheme $(n=16)$.

\begin{tabular}{|c|c|c|c|c|c|c|}
\hline \multirow{2}{*}{ Scheme } & \multicolumn{2}{|c|}{$E_{\text {total }}<0.9823[u l p]$} & \multicolumn{2}{|c|}{$E_{\text {total }}<0.7321[u l p]$} & \multicolumn{2}{|c|}{$E_{\text {total }}<0.6070[u l p]$} \\
\cline { 2 - 8 } & $\mathrm{k}$ & Area [gates] & $\mathrm{k}$ & Area [gates] & $\mathrm{k}$ & Area [gates] \\
\hline CCT & 3 & $1712(100 \%)$ & 4 & $1840(100 \%)$ & 5 & $1950(100 \%)$ \\
\hline VCT & 3 & $1803(+4.6 \%)$ & 3 & $1803(-2.0 \%)$ & 4 & $1921(-1.5 \%)$ \\
\hline PCT & 2 & $1644(-3.9 \%)$ & 3 & $1754(-4.6 \%)$ & 4 & $1883(-3.4 \%)$ \\
\hline Proposed & 1 & $1531(-11.0 \%)$ & 2 & $1660(-9.8 \%)$ & 3 & $1774(-9.0 \%)$ \\
\hline
\end{tabular}

Table II. PSNR (dB) comparison for DCT/IDCT image processing.

\begin{tabular}{|l|c|c|c|}
\hline \multirow{2}{*}{ Scheme } & \multicolumn{3}{|c|}{$k$} \\
\cline { 2 - 4 } & 1 & 2 & 3 \\
\hline CCT (hardware) & 24.43 & 28.32 & 30.92 \\
VCT (hardware) & 29.72 & 34.05 & 37.66 \\
PCT (hardware) & 29.34 & 33.89 & 37.83 \\
Proposed (hardware) & 33.25 & 38.35 & 41.73 \\
\hline Direct Truncated (hardware) & \multicolumn{3}{|c|}{20.26} \\
Floating-point (software) & \multicolumn{3}{|c|}{46.47} \\
\hline
\end{tabular}

Table III. PSNR (dB) comparison for image CSC processing.

\begin{tabular}{|l|c|c|c|}
\hline \multirow{2}{*}{ Scheme } & \multicolumn{3}{|c|}{$k$} \\
\cline { 2 - 4 } & 1 & 2 & 3 \\
\hline CCT (hardware) & 27.80 & 30.53 & 31.82 \\
VCT (hardware) & 30.02 & 32.35 & 34.77 \\
PCT (hardware) & 29.73 & 32.08 & 34.58 \\
Proposed (hardware) & 32.25 & 34.35 & 36.73 \\
\hline Direct Truncated (hardware) & \multicolumn{3}{|c|}{22.17} \\
Floating-point (software) & 38.35 \\
\hline
\end{tabular}

To exhibit the accuracy of the proposed scheme in real applications, we 
$8 \times 82$-D DCT and IDCT processing cores are built by using $8 \times 8$-bit truncated multipliers. The test image (512 pixel $\times 512$ pixel "Lena") is first compressed by using the DCT core and then decompressed back by using the IDCT core. For CSC, a test image in $4: 4: 4$ RGB format is first transformed into $\mathrm{YCbCr}$ color space (4:2:0 format) and then transformed back to the original format. Multiplication by constant coefficients are implemented by using truncated multipliers instead of a distributed arithmetic scheme based on look-up table [10]. In both applications, direct truncated, CCT, VCT, PCT and OPEB-CCT multipliers are tested with the parameters set to $k=1,2,3$. The accuracy performance is evaluated based on the peak signal-to-noise ratio (PSNR). Software based (i.e., using floating-point arithmetic) results are also listed for reference. The measured results are reported in Tables II and III.

Compared with the PCT scheme, which has the best performance among the reference schemes, the proposed approach achieves an average PSNR improvement of $4.09 \mathrm{~dB}$ for DCT/IDCT application and $2.31 \mathrm{~dB}$ for CSC application, respectively. Compared with directly truncated multiplication, the proposed design with the lowest hardware cost $(\mathrm{k}=1)$ improves the PSNR by $12.99 \mathrm{~dB}$ and $10.08 \mathrm{~dB}$. The results also show that to achieve the same PSNR performance, the reference schemes generally have to use a larger $k$, which is consistent with the experimental results presented in the previous section.

\section{Conclusion}

In this brief, a reduced-error CCT multiplier design scheme is proposed. By correctly computing the probability of occurrence of the input operands, partial and final product bits, the proposed scheme estimates the reduction and rounding errors more accurately than traditional schemes. Therefore, the proposed scheme can reduce the computation error by applying a more appropriate correction constant. $8 \times 8$-bit and $16 \times 16$-bit truncated array multipliers are finally implemented in $65 \mathrm{~nm}$ standard cell technology and tested in real image compression and color space conversion applications. Experimental results show that the proposed scheme achieves a $4.09 \mathrm{~dB}$ PSNR improvement in the DCT/IDCT based image compression application and a $2.31 \mathrm{~dB}$ PSNR improvement for the CSC application over the PCT multipliers. The proposed scheme can also achieve 5.8\% hardware cost reduction over the PCT scheme when the same truncation error performance is desired. Therefore, the proposed reduced-error CCT multiplier is an ideal choice for cost-aware digital signal processing and multimedia processing designs of integrated circuits and microchips.

\section{Acknowledgments}

This work was supported by the National Natural Science Foundation of China Grant No. 61106022 and Beijing Natural Science Foundation NO. 4143066. 